University of South Carolina

Scholar Commons

$6-1-2003$

\title{
Thermal Management of AIGaN-GaN HFETs on Sapphire Using Flip-Chip Bonding with Epoxy Underfill
}

Jie Sun

University of South Carolina - Columbia, sunjie@engr.sc.edu

H. Fatima

University of South Carolina - Columbia

Alexei Koudymov

University of South Carolina - Columbia

A. Chitnis

University of South Carolina - Columbia

X. Hu

SET, Inc.

See next page for additional authors

Follow this and additional works at: https://scholarcommons.sc.edu/elct_facpub

Part of the Electrical and Computer Engineering Commons

Publication Info

Published in IEEE Electron Device Letters, Volume 24, Issue 6, 2003, pages 375-377.

http://ieeexplore.ieee.org/xpl/Recentlssue.jsp?punumber=55

(C) 2003 by IEEE

This Article is brought to you by the Electrical Engineering, Department of at Scholar Commons. It has been accepted for inclusion in Faculty Publications by an authorized administrator of Scholar Commons. For more information, please contact digres@mailbox.sc.edu. 


\section{Author(s)}

Jie Sun, H. Fatima, Alexei Koudymov, A. Chitnis, X. Hu, H.-M Wang, J. Zhang, Grigory Simin, J. Yang, and Asif Khan 


\title{
Thermal Management of AlGaN-GaN HFETs on Sapphire Using Flip-Chip Bonding With Epoxy Underfill
}

\author{
Jie Sun, Student Member, IEEE, H. Fatima, A. Koudymov, A. Chitnis, Member, IEEE, X. Hu, Member, IEEE, \\ H.-M. Wang, J. Zhang, G. Simin, Member, IEEE, J. Yang, and M. Asif Khan, Senior Member, IEEE
}

\begin{abstract}
Self-heating imposes the major limitation on the output power of GaN-based HFETs on sapphire or SiC. SiC substrates allow for a simple device thermal management scheme; however, they are about a factor 20-100 higher in cost than sapphire. Sapphire substrates of diameters exceeding 4 in are easily available but the heat removal through the substrate is inefficient due to its low thermal conductivity. The authors demonstrate that the thermal impedance of GaN based HFETs over sapphire substrates can be significantly reduced by implementing flip-chip bonding with thermal conductive epoxy underfill. They also show that in sapphire-based flip-chip mounted devices the heat spread from the active region under the gate along the $\mathrm{GaN}$ buffer and the substrate is the key contributor to the overall thermal impedance.
\end{abstract}

Index Terms-Epoxy underfill, flip chip, GaN AlGaN, HFETs, thermal impedance.

\section{INTRODUCTION}

A LGAN-GaN heterostructure field effect transistors (HFETs) [1], [2] have demonstrated great microwave power performance due to the high-breakdown field and the high-electron saturation velocity. For HFETs, the GaN-AlGaN epitaxial layers have been grown on either sapphire or $\mathrm{SiC}$ substrates. Although sapphire has the advantage of lower cost and availability in larger wafer sizes, its poor thermal conductivity $(\sigma \approx 0.3 \mathrm{~W} / \mathrm{cm}-\mathrm{K}$ ) limits the achievable powers due to severe self-heating. The thermal impedance can be significantly reduced by flip-chip (FC) mounting the devices onto highly conducting substrates such as $\operatorname{AlN}(\sigma=1.80 \mathrm{~W} / \mathrm{cm}-\mathrm{K})$. Such an FC mounting scheme has been demonstrated to achieve high-output power GaN HFETs on sapphire [3]-[5]. These past works used gold bump bonding and AlN carrier for device thermal management. However, no data was presented comparing devices with and without FC. In addition, there was

Manuscript received February 10, 2003. This work was supported in part by the U.S. Army under SMDC Grant DASG60-00-10003, monitored by T. Bauer, and by the Defense Advanced Research Project Agency under Grant DAAD19-02-10 236, monitored by Dr. E. Martinez and Dr. A. Hung. The work at Sensor Electronic Technology (SET), Inc., was supported by the Missile Defense Agency under SBIR Contract F33615-01-C-1911, monitored by G. Gillispie of Wright-Patterson AFB, and under U.S. Army SMDC Contract DASG60-00-C-0088, monitored by F. Clarke. The review of this letter was arranged by Editor D. Ritter.

J. Sun, H. Fatima, A. Koudymov, A. Chitnis, H.-M. Wang, J. Zhang, G. Simin, J. Yang, and M. A. Khan are with the Department of Electrica Engineering, University of South Carolina, Columbia, SC 29208 USA (e-mail: sunjie@engr.sc.edu).

X. Hu is with SET, Inc., Columbia, SC 29209 USA.

Digital Object Identifier 10.1109/LED.2003.813362 no discussion of the thermal impedance value and the effect of device design parameters on it. In this paper, we now present a novel thermal management approach for GaN HFETs over sapphire, which uses an FC technique with thermoconductive epoxy underfill. We demonstrate our approach to be effective for controlling the heat dissipation from the active area of device to the AlN substrate. We also show that the heat spread along the chip contributes significantly into the total thermal impedance of FC devices.

\section{FC HFETS DESIGN, FABRICATION, AND CHARACTERIZATION}

The HFET epilayers structures for our study were grown over basal plane sapphire or insulating $\mathrm{SiC}$ substrates using the low-pressure metal organic chemical vapor deposition (MOCVD) technique. The $\mathrm{AlGaN}-\mathrm{GaN}$ layers were deposited at $1000{ }^{\circ} \mathrm{C}$ and 76 torr. The growth details were published elshwere [6]. The ohmic contacts for source and drain were fabricated using e-beam deposited $\operatorname{Ti}(200 \mathrm{~A})-\mathrm{Al}(500 \mathrm{~A})-\operatorname{Ti}(200 \mathrm{~A})-\mathrm{Au}(1500 \mathrm{~A})$. They were annealed at $850{ }^{\circ} \mathrm{C}$ for $1 \mathrm{~min}$ in nitrogen ambient. $\mathrm{Ni}(200 \mathrm{~A})-\mathrm{Au}(1000 \mathrm{~A})$ gate electrodes were then deposited. The devices had a U-shaped double gate-finger geometry with a single gate length and width of 1.5 and $100 \mu \mathrm{m}$, respectively. Reactive ion etched mesas were used for device-to-device isolation. The devices were then FC bonded onto 500- $\mu$ m-thick AlN substrate using gold bumps. No-flow epoxy underfill was then used to fill the air gap between the chip and the AlN substrate with thermoconductive material. Ours is the first report of using epoxy underfill for FC GaN based HFETs.

For our experiments, an automated FC die bonder RD M-10A system and thermoconductive boron nitride epoxy with low coefficient of thermal expansion (CTE) was used. The alignment accuracy of the RD system is within $\pm 1 \mu \mathrm{m}$. No flow underfill (controlled volume epoxy) is applied on the substrate through an advanced syringe dispensing system. The charge-coupled device (CCD) images of FC samples show that the bumps were aligned within $\sim 6 \mu \mathrm{m}$ from the gate electrode. The bumps' diameter and the height (after bonding) were about 120 and $10 \mu \mathrm{m}$, respectively.

For our experimental study of lateral heat spreading along the chip, we selected HFET devices on sapphire and $\mathrm{SiC}$ substrates with nearly identical peak currents (measured in pulsed mode to avoid the self-heating). The HFETs over sapphire substrates were also FC mounted with and without epoxy underfill. 


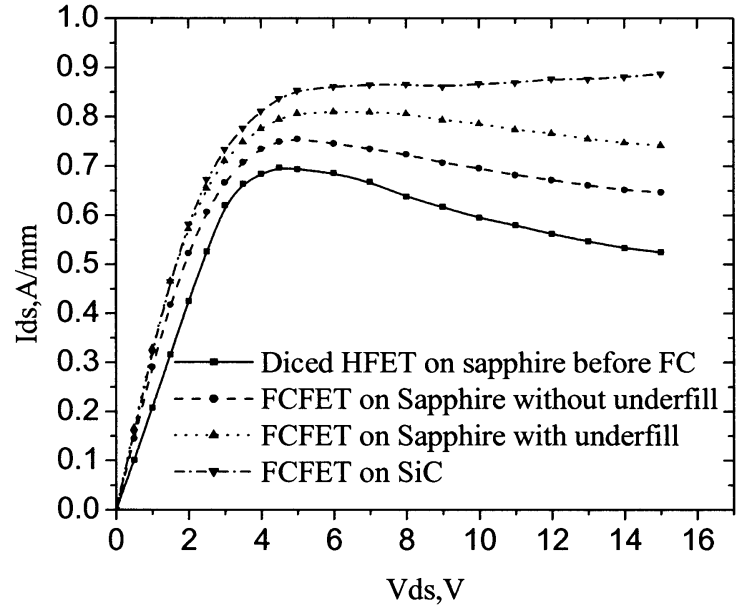

Fig. 1. DC $I-V$ characteristics of HFETs over sapphire at $V_{g}=0 \mathrm{~V}$ after dicing, after FC bonding, after FC with epoxy underfill. Also shown is the $I-V$ for FC HFET grown over SiC substrate.

TABLE I

THERMAL IMPEDANCE OF THE FC HFETs

\begin{tabular}{c|c}
\hline Device packaging configuration & Thermal impedance \\
\hline Diced HFET on sapphire before FC & $51.7 \mathrm{~K}-\mathrm{mm} / \mathrm{W}$ \\
\hline FC FET on sapphire without underfill & $22.4 \mathrm{~K}-\mathrm{mm} / \mathrm{W}$ \\
\hline FC FET on sapphire with underfill & $16.5 \mathrm{~K}-\mathrm{mm} / \mathrm{W}$ \\
\hline FC FET on SiC & $4.9 \mathrm{~K}-\mathrm{mm} / \mathrm{W}$ \\
\hline
\end{tabular}

DC current-voltage $I-V$ characteristics at zero gate bias for the different devices of this study are shown in Fig. 1. The $I-V$ 's before and after FC without underfill are shown for the same device, other $I-V$ 's are shown for devices with identical pulse peak currents. As seen, the FC bonding results in dramatic improvement of the dc- $I-V$ characteristics. The threshold voltage and the gate leakage current practically did not change after the FC process.

The thermal impedance $R_{\text {th }}$ of FC devices was then extracted by a comparison of the pulsed (200-ns pulse width, $0.05 \%$ duty cycle) and the dc $I-V$ characteristics at different ambient temperatures. These values for the various packaging configuration in our study are summarized in Table I.

\section{DISCUSSION}

Schematic layout of an FC HFET device with gold bumps and epoxy underfill is shown in Fig. 2(a). The gold bumps $(\sigma=3 \mathrm{~W} / \mathrm{cm}-\mathrm{K})$ serve as paths for heat dissipation as well as low-inductance connection. A simplified equivalent thermal impedance "network" for this design is shown in Fig. 2(b). Here, $R_{\text {th1 }}$ represents the heat flux through epoxy underfill region, $R_{\mathrm{th} 2}$ is the impedance due to lateral heat spread along the chip, and $R_{\mathrm{th} 3}$ is the thermal impedance of the gold bumps.

For the lateral heat flow in the HFET chip, one has to take into account the nonuniform heat spread in the bump area [see Fig. 2(a)]. This problem is very similar to the lateral electrical current spreading in the contact area as analyzed in [7]. Using

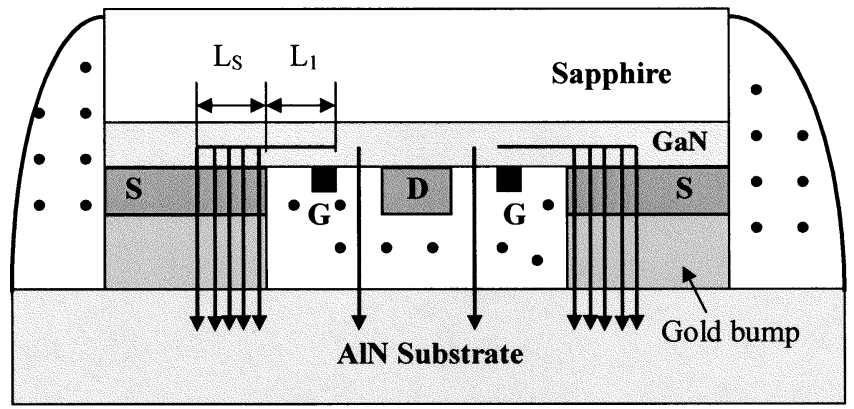

(a)

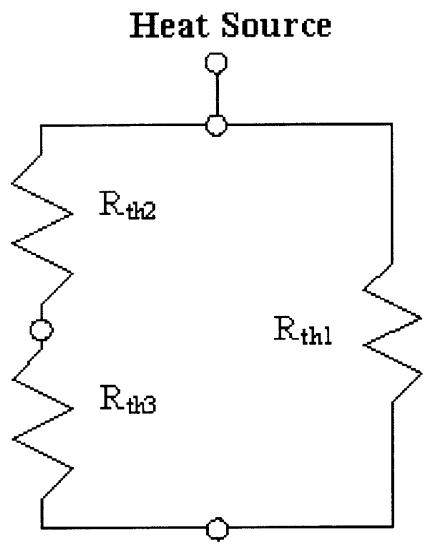

AIN substrate

(b)

Fig. 2. (a) Schematics of the FC bonded AlGaN-GaN HFETs with epoxy underfill. (b) Simplified thermal impedance network.

a thermo-electrical analogy and the approach developed in [7], we can estimate the effective heat-spreading length

$$
L_{\mathrm{S}}=\sqrt{\frac{\sigma_{2}}{\sigma_{3}}} \cdot d \cdot h
$$

where the thermal conductivities of $\mathrm{GaN}$ and the bump are, respectively, $\sigma_{2} \approx 1.6 \mathrm{~W} /(\mathrm{cm}-\mathrm{K})$ and $\sigma_{3} \approx 3 \mathrm{~W} /(\mathrm{cm}-\mathrm{K}), d$ is the thickness of GaN buffer, and $h \approx 10 \mu \mathrm{m}$ is the bump height. The lateral component of thermal impedance $R_{\mathrm{th} 2}$ can, therefore, be estimated as $R_{\mathrm{th} 2}=\left(1 / \sigma_{2}\right) \times\left(L_{1}+L_{S}\right) / d$ [please refer to Fig. 2(a)]. For our sample $d \approx 1.5 \mu \mathrm{m}$ and $h \approx 10 \mu \mathrm{m}$, thus from (1) we find $L_{S} \approx 2.8 \mu \mathrm{m}$. Noting the gold-bump width to be $120 \mu \mathrm{m}$, we conclude that only the edge of the bump contributes significantly into heat removal from the chip. Using $L_{1} \sim L_{G}+L_{\mathrm{GS}} \approx 2.5 \mu \mathrm{m}$, where $L_{G} \approx 1 \mu \mathrm{m}$ and $L_{\mathrm{GS}} \approx 1.5 \mu \mathrm{m}$ are the gate length and the gate-source spacing, respectively, we estimate $R_{\mathrm{th} 2} \approx 22.1 \mathrm{~K}-\mathrm{mm} / \mathrm{W}$. For a $5-\mu$ $\mathrm{m}$-thick buffer $R_{\mathrm{th} 2}$ can be as low as $16 \mathrm{~K}-\mathrm{mm} / \mathrm{W}$. The thermal impedance of the gold bumps can be found as follows [8]:

$$
R_{\mathrm{th} 3}=\frac{1}{\pi \sigma_{3}} \ln \left(\frac{16 h}{\pi L_{S}}\right) .
$$

This results in $R_{\mathrm{th} 3} \approx 3.1 \mathrm{~K}-\mathrm{mm} / \mathrm{W} \ll R_{\mathrm{th} 2}$. Therefore, the lateral heat spread along the HFET chip is the limiting factor in the overall thermal impedance of the FC samples. This analysis, therefore, shows GaN buffer thickness to be the key controller of 
lateral thermal impedance and hence the thermal management of FC devices over sapphire [9]. This conclusion is clearly supported by the experimental data of Table I.

The value of $R_{\mathrm{th} 1}$ associated with epoxy filling can be estimated from (2), replacing $\sigma_{3}$ with the epoxy thermoconductivity $\sigma_{1} \approx 0.05 \mathrm{~W} /(\mathrm{cm}-\mathrm{K})$, and $L_{S}$ with $L_{0}$, where $L_{0}$ is the effective width of the heat source. Using (1) we can see that for GaN-epoxy interface $L_{S} \approx 20 \mu \mathrm{m}$, i.e., much larger than the entire source-drain spacing, $L_{\mathrm{DS}}=5 \mu \mathrm{m}$ for our samples. Therefore, considering the heat flow through epoxy undefill, the effective length of the heat source is about $L_{0} \approx L_{\mathrm{DS}}=5 \mu \mathrm{m}$. From (2), we can now find the thermal impedance associated with epoxy underfill, $R_{\mathrm{th} 1} \approx 140 \mathrm{~K}-\mathrm{mm} / \mathrm{W}$. We can see that epoxy underfill improves the total thermal impedance by about $20 \%$. This agrees quite well with the experimental data of Table I for the FC devices with and without epoxy underfilling (lines 2 and 3). The RF performance analysis of the FC devices is undergoing; however, a simple estimate shows that with a typical $40 \%$ PAE in class A mode, the maximum active region temperature of $250{ }^{\circ} \mathrm{C}$ and $16.5 \mathrm{~K}-\mathrm{mm} / \mathrm{W}$ thermal impedance the RF power of $\sim 6 \mathrm{~W} / \mathrm{mm}$ can be achieved using FC devices.

\section{CONCLUSION}

In conclusion, we report the thermal management of $\mathrm{AlGaN}-\mathrm{GaN}$ HFETs on sapphire by FC bonding with epoxy underfill. We show the FC bonding procedure not to affect the device performance parameters. Compared to a diced chip, the thermo-compression gold bump FC bonding with epoxy underfill decreases the thermal impedance of HFETs over sapphire by a factor of three. Our experimental data and heat spread analysis show that the thermal impedance can be further reduced by increasing the $\mathrm{GaN}$ buffer layer thickness.

\section{REFERENCES}

[1] M. A. Khan, J. M. Van Hove, J. N. Kuznia, and D. T. Olsen, "High electron mobility GaN/AlGaN heterostructures grown by LPMOCVD," Appl. Phys. Lett., vol. 58, pp. 2408-2410, 1991.

[2] M. A. Khan, A. Bhattarai, J. N. Kuznia, and D. T. Olson, "High electron mobility transistor based on $\mathrm{GaN}-\mathrm{Al}_{x} \mathrm{Ga}_{1-x} \mathrm{~N}$ heterojunction," Appl. Phys. Lett., vol. 63, pp. 1214-1215, 1993.

[3] Y.-F. Wu, D. Kapolnek, J. P. Ibbetson, P. Parikh, B. P. Keller, and U. K. Mishra, "Very-high power density AlGaN/GaN HEMTs," IEEE Trans. Microwave Theory Tech., vol. 48, pp. 586-590, 2001.

[4] J. J. Xu, Y.-F. Wu, S. Keller, G. Parish, S. Heikman, B. J. Thibeault, U. K. Mishra, and R. A. York, "1-8-GHz GaN-based power amplifier using flip-chip bonding," IEEE Microwave Guided Wave Lett., vol. 9, pp. 277-279, 1999.

[5] J. J. Xu, S. Keller, G. Parish, S. Heikman, U. K. Mishra, and R. A. York, "A 3-10-GHz GaN-based flip-chip integrated broad-band power amplifier," IEEE Trans. Microwave Theory Tech., vol. 48, pp. 2573-2578, 2000.

[6] M. A. Khan, X. Hu, G. Simin, A. Lunev, J. Yang, R. Gaska, and M. S. Shur, "AlGaN/GaN metal-oxide-semiconductor heterostructure field effect transistor," IEEE Electron Device Lett., vol. 21, pp. 63-65, Jan. 2000.

[7] M. Shatalov, J. P. Zhang, A. Chitnis, V. Adivarahan, J. W. Yang, G. Simin, and M. A. Khan, "Deep ultraviolet light emitting diodes using quaternary AlInGaN multiple quantum wells," IEEE J. Select. Topics Quantum Electron., vol. 8, pp. 302-309, 2002.

[8] J. V. Dilorenzo and D. D. Khandelwal, GaAs FET Principles and Technology. Norwell, MA: Artech House, 1982, pp. 335-336.

[9] R. Gaska, A. Osinsky, J. W. Yang, and M. S. Shur, "Self-heating in high power AlGaN/GaN HFETs," IEEE Electron. Device Lett., vol. 19, pp. 89-91, Feb. 1998. 